\title{
Condición física y calidad de vida de mujeres privadas de libertad del centro penitenciario de Concepción
}

\author{
Physical condition and quality of life of women deprived of liberty in \\ the Concepción penitentiary
}

\author{
* Yazmina Virginia Pleticosic Ramírez \& ** Mariangel Javiera Correa Recabal
}

Pleticosic, Y., \& Correa, M. (2021). Condición física y calidad de vida de mujeres privadas de libertad del centro penitenciario de Concepción. Revista Ciencias de la Actividad Física UCM, № 22(1), enero-junio, 1-11. DOI: http://doi.org/10.29035/rcaf.22.1.5

\begin{abstract}
RESUMEN
El presente estudio, tuvo como objetivo determinar la condición física, el estado nutricional, el riesgo cardiovascular y la calidad de vida de mujeres privadas de libertad (MPL) del centro penitenciario de Concepción de la región del Biobío. El estudio fue de diseño no experimental-transversal de alcance descriptivo. La condición física fue valorada a través de las siguientes pruebas; test Sit and Reach para flexibilidad, CMJ para la fuerza explosiva del tren inferior y la prueba del escalón de 3 minutos para resistencia aeróbica. Para valorar la calidad de vida se utilizó la WHOQoLBref. Además, se calculó IMC e ICC, a través de la medición de la talla, peso y perímetro de cintura, utilizando una balanza con tallímetro (Seca 220) y cinta métrica Lufkin. Las mujeres privadas de libertad presentaron un promedio de 39,58 \pm 15,30 años de edad, un IMC de 27,85 \pm 6,08 kg/m², y un ICC de 0,85 \pm 0,05. Los valores promedios para la flexibilidad fueron de 35,91 $\pm 11,61 \mathrm{~cm}$, para CMJ fue 13,46 \pm 4,72 cm y en el test del escalón 28,32 \pm 5,48 de eficiencia cardiovascular. La percepción de la calidad de vida general fue mala $(2,41 \pm 0,66)$ y las dimensiones de salud física, psicológica, relaciones sociales y ambiente se posicionan en el percentil más bajo (P5).
\end{abstract}

Palabras clave: Condición física, calidad de vida, composición corporal, reclusas.

\begin{abstract}
The objective of the present study was to determine the physical condition, nutritional status, cardiovascular risk and quality of life of women deprived of liberty (MPL) from the Concepción penitentiary in the Biobío region. The study was of a non-experimental-cross-sectional design with a descriptive scope. The physical condition was assessed through the following tests; Sit and Reach test for flexibility, CMJ for explosive lower body strength and the 3-minute step test for aerobic endurance. To assess quality of life, the WHOQOL-Bref was used. In addition, BMI and ICC were calculated by measuring height, weight and waist circumference, using a scale with a height rod (Seca 220) and a Lufkin tape measure. The women deprived of liberty presented an average age of $39.58 \pm 15.30$ years, a $\mathrm{BMI}$ of $27.85 \pm 6.08 \mathrm{~kg} / \mathrm{m}^{2}$, and an ICC of $0.85 \pm 0.05$. The mean values for flexibility were $35.91 \pm 11.61 \mathrm{~cm}$, for CMJ it was $13.46 \pm 4.72 \mathrm{~cm}$ and test step $28.32 \pm 5.48$ of cardiovascular efficiency. The perception of the general quality of life was poor ( $2.41 \pm 0.66$ ) and the dimensions of physical, psychological, social relations and environment were in the lowest percentile (P5).
\end{abstract}

Key words: Physical condition, quality of life, body composition, inmates. 


\section{INTRODUCCIÓN}

La población penal femenina en Chile privativa de libertad con régimen cerrado, alcanza un total de 3.519 y ocupa el $2^{\circ}$ lugar en América del Sur, con un $8,4 \%$. En la región del Biobío, las mujeres privadas de libertad representan el 7,2\% de la población penal (Gendarmería de Chile, 2018). "La prisión es para la mujer un espacio discriminador y opresivo, particularmente por el significado que asume el encierro. Se trata de una experiencia doblemente estigmatizadora y dolorosa, dado el rol que la sociedad le ha asignado" (Cárdenas, 2011, p.5). El perfil de las reclusas corrobora que se trata de mujeres pobres, con bajo nivel educacional, que participan en delitos menores, que son madres, jefas de hogar, y el principal soporte familiar (Casas et al., 2005).

La protección y reconocimiento en materia de derechos humanos, no ha cubierto con igual intensidad a personas que se encuentran en el sistema privativo de libertad, que las hace más vulnerables, en especial a las mujeres que con frecuencia llegan a la prisión con una serie de problemas de salud no tratados (Instituto Nacional de Derechos Humanos [INDH], 2012). "El sistema penitenciario chileno tiene una serie de dificultades que impiden a las reclusas y reclusos, acceder a prestaciones de salud básicas y en los tiempos requeridos de acuerdo a sus necesidades" (Vargas, 2019, p. 535). El derecho a la salud implica tanto libertades como derechos, dentro de las libertades se encuentra controlar la salud y el cuerpo y dentro de los derechos se encuentran los relativos a un sistema que proteja la salud otorgando oportunidades igualitarias para disfrutar del nivel más alto de salud que se pueda alcanzar (Comité de Derechos Económicos Sociales y Culturales, 2000). El abandono gubernamental en materia de salud dentro de la cárcel vuelve a la mujer más vulnerable dentro y fuera de ella, además se observa que las reclusas sufren de una mayor vulnerabilidad física y emocional en comparación con los hombres (Cárdenas, 2011) y diversos factores pueden modificar su calidad de vida (Robles et al., 2016).
La Organización Mundial de la Salud define la calidad de vida como las percepciones de los individuos sobre su posición en la vida en el contexto de la cultura y los sistemas de valores en los que viven y en relación con sus objetivos, expectativas, estándares y preocupaciones. Es un concepto de amplio alcance que incorpora de manera compleja la salud física, el estado psicológico, el nivel de independencia, las relaciones sociales, las creencias personales y sus relaciones de las personas para enfatizar las características del entorno (World Health Organization, 1998, p. 3).

El bienestar físico y psicológico son indisolubles, los trastornos psicológicos influencian la aparición de enfermedades físicas crónicas como las cardiovasculares, cáncer, diabetes u obesidad (Gómez, 2007). En función del alcance físico y psicológico, se puede decir que "el estado de salud puede variar dependiendo de cuál sea su estado anímico... las personas que presentan un estado anímico negativo están más predispuestas a interpretar un indicio fisiológico como un posible problema de salud" (Barra, 2003, p. 56).

En la última Encuesta Nacional de Salud (2017) realizada en Chile, las mujeres presentaron un 90,0\% de sedentarismo, un $36,4 \%$ de sobrepeso y un $33,7 \%$ de obesidad. El 27,7 \% de las mujeres tiene hipertensión, un 14,0\% tiene diabetes mellitus, un 2,8\% autoreporta infarto agudo al miocardio y un 3,2\% ACV. Los niveles de obesidad de las mujeres chilenas adultas lideran dentro de la población femenina de Sudamérica. El sobrepeso y la obesidad representan un riesgo para la salud, debido a que está asociado a algunas enfermedades no transmisibles (ECNT) (Organización de las Naciones Unidas para la Alimentación y la Agricultura [FAO] y Organización Panamericana de la Salud [OPS], 2017). Evidencia científica muestra que las personas físicamente inactivas tienen más riesgo de morir por ECNT (OMS, 2010). Ding et al. (2016) citado en Celis et al. (2016) concluyeron que, si las personas en Chile cumplieran con las 
recomendaciones mínimas de actividad física "el riesgo de ECNTs atribuible a inactividad física disminuiría en un 3,5\% para enfermedad coronaria, 4,0\% para enfermedad cerebrovascular, 4,4\% para diabetes mellitus tipo 2, 6,5\% para cáncer de mamas, 6,3\% para cáncer de colon y 5,7\% para mortalidad general" (p. 1092). En este sentido "pocos trabajos han tenido la posibilidad de analizar y presentar datos nacionales sobre la situación de las mujeres encarceladas o de indagar sobre sus necesidades de intervención" (Sanhueza et al., 2019, p. 120). Por lo que resulta importante conocer la realidad de la mujer chilena privada de libertad en función de su condición física, riesgo cardiovascular, estado nutricional y calidad de vida dentro de la penitenciaria. "La condición física (CF) es un importante indicador fisiológico del estado de salud de la población en general" (Rosa et al.,

\section{DISEÑO METODOLÓGICO}

El estudio fue de diseño no experimental-transversal de alcance descriptivo (Hernández et al., 2014). La muestra se constituyó de 12 mujeres adultas privadas de libertad del Centro Penitenciario de Concepción de la región del Biobío, Chile. Sus edades fluctuaron entre 23 y 65 años. Se utilizó un muestreo no probabilístico por conveniencia (Otzen \& Manterola, 2017). Todas las mujeres que accedieron a participar firmaron un consentimiento informado.

Los criterios de inclusión fueron: mujeres mayores de edad de permanencia estable o transitoria en el centro de penitenciario que quisieran participar de la investigación y autorización médica del centro penitenciario. En cuanto a los criterios de exclusión fueron: mujeres que presentarán problemas físicomotores para rendir las pruebas de la condición física; mujeres que no pudieran finalizar los protocolos de evaluación de la condición física; mujeres que no firmarán el consentimiento informado.

Se informó previamente al centro penitenciario como a las MPL acerca del objetivo del estudio. Los instrumentos de evaluación fueron ingresados al centro penitenciario con la debida autorización de Gendarmería de Chile y con antelación a los días de evaluación. En función del tiempo de contacto con
2017, p. 37), permite la obtención de información acerca del estado de salud y la calidad de vida, aspectos importantes a tener en cuenta al momento de atender programas de actividad física y salud, como también necesarios para orientar programas generales que promocionan la salud (Zaragoza et al., 2004).

Debido a la escasa investigación acerca de las condiciones de salud y calidad de vida actuales de la población penal femenina en Chile y su relevancia para la situación penitenciaria del país, el objetivo del presente estudio fue determinar la condición física, el estado nutricional, el riesgo cardiovascular y la calidad de vida de mujeres privadas de libertad (MPL) del centro penitenciario de Concepción de la región del Biobío.

las MPL y las actividades diarias del centro penitenciario, las evaluaciones se llevaron a cabo en 3 sesiones de 60 minutos durante el mes de agosto del año 2019. En la primera sesión se completó la encuesta de calidad de vida y las medidas antropométricas; la segunda y la tercera sesión se realizaron las evaluaciones de la condición física.

La valoración del estado nutricional y riesgo cardiovascular se estimó a través del IMC e ICC, para ello se registró la talla, peso, perímetro de cintura y cadera, utilizando una balanza con tallímetro (Seca 220) y cinta métrica Lufkin.

La condición física fue valorada a través de los siguientes protocolos: Sit and Reach para evaluar la flexibilidad de la zona lumbar y cadera, donde se marca con una cinta de medir de manera recta y vertical desde los 0 a $38 \mathrm{~cm}$. El evaluado sentado en el suelo apoya los talones al ancho de los hombros y allí extiende ambos brazos y manos inclinando el tronco hacia delante hasta el máximo que le permita su flexión de tronco, sin doblar las rodillas, se realizan dos intentos y se registra el mejor resultado (American College of Sports Medicine Instituto [ACSM], 2005); CMJ, test de salto en contra movimiento que evalúa la fuerza explosiva del tren 
inferior, con tres intentos seleccionando el mejor resultado alcanzado (Bosco, 1996). Se utilizó la plataforma de salto DMJump®; Prueba del escalón de 3 minutos para medir el nivel de eficiencia cardiovascular, una vez concluida la prueba, el sujeto descansa 1 minuto y se registra la frecuencia cardiaca (Martínez, 2002). Se utilizó un monitor de frecuencia cardiaca marca Polar modelo V800 HR para medir la frecuencia cardiaca de recuperación.

Para la valoración de la calidad de vida se les aplicó la encuesta de Calidad de Vida de la Organización Mundial de la Salud, modalidad abreviada WHOQoL-Bref, que evalúa 4 dimensiones: salud

\section{RESULTADOS}

Se evaluaron 12 mujeres privadas de libertad del Centro Penitenciario de Concepción de la región del Biobío. Se realizaron mediciones antropométricas, pruebas de condición física y aplicación de un cuestionario de calidad de vida. La edad promedio presentada por las MPL fue de 39,58 \pm 15,30 años. La Tabla 1 muestra los resultados antropométricos y de condición física obtenida de la muestra total.

El IMC es uno de los métodos más recomendados para determinar el exceso de peso y obesidad en población sedentaria (Mei et al., 2002). Las MPL presentan un IMC promedio categorizada en sobrepeso física, salud psicológica, relaciones sociales y ambiente (OMS, 1996) con valores de referencia para población adulta chilena (Urzúa \& Caqueo, 2013). El instrumento WHOQOL-Bref ofrece un perfil de calidad de vida, siendo cada dimensión o dominio puntuado de forma independiente. Cuanto mayor sea la puntuación en cada dominio, mejor es el perfil de calidad de vida de la persona evaluada (Bobes et al., 2004).

El análisis estadístico y la tabulación de los datos se realizó a través del paquete Microsoft Excel $2013 \AA$.

$(27,85 \pm 6,08)$ (OMS, 1995). El ICC promedio indica un riesgo cardiovascular moderado $(0,85 \pm 0,05)$ para mujeres adultas (Ministerio de Salud [MINSAL], 2008).

En función de las pruebas para evaluar la condición física tenemos que en el test del escalón las MPL presentan un índice de aptitud cardiorrespiratoria promedio de $28,32 \pm 5,48$. En la prueba de salto (CMJ) el promedio fue de 13,46 $\pm 4,72 \mathrm{~cm}$ y en la prueba de flexibilidad el promedio fue de 35,91 \pm 11,61 $\mathrm{cm}$.

Tabla 1

Resultados antropométricos y de condición física de las MPL.

\begin{tabular}{lcccc}
\hline Evaluaciones & MPL (nT2) & DE & Min & Máx \\
\hline Edad (años) & M & & 23 & 65 \\
Índice masa corporal (Kg/m²) & 27,58 & 15,30 & 20,3 & 40,1 \\
Índice cintura-cadera (ICC) & 0,85 & 6,08 & 0,76 & 0,94 \\
Sit and Reach (cm) & 35,91 & 0,05 & 15 & 48 \\
CMJ (cm) & 13,46 & 11,61 & 4 & 19 \\
Test del escalón (IAC) & 28,32 & 4,72 & 22,16 & 37,37 \\
\hline
\end{tabular}

Fuente: elaboración propia.

Para valorar la calidad de vida se utilizó el cuestionario WHOQoL-Bref. En la Tabla 2, se presentan los promedios de las puntuaciones obtenidas en cada ítem que componen el cuestionario y en la Tabla 3 se muestran los resultados obtenidos por dimensiones con la puntuación de 0 a 100 para comparar con los valores de referencias del WHOQOL- 
Bref en población adulta chilena (Urzúa \& Caqueo, 2013)

En la primera pregunta del cuestionario, acerca de la percepción de la calidad de vida general, el promedio de respuestas de las MPL fue categorizada como mala $(2,41 \pm 0,66)$. La segunda, respecto a la satisfacción de las MPL con la salud, obtienen un promedio que se categoriza como un poco satisfecho $(2,5 \pm 1,16)$. Los resultados promedios en salud física, psicológica, relaciones sociales y ambiente para la edad, se posiciona en el percentil 5, al comparar con los valores de referencia del WHOQOL-Bref para población adulta chilena (Urzúa \& Caqueo, 2013).

Tabla 2

Resultados cuestionario de calidad de vida (WHOQoL-Bref) de las MPL por ítem.

\begin{tabular}{|c|c|c|c|c|}
\hline Ítem WHOQoL-Bref & $\begin{array}{c}\text { General }(n=12) \\
M\end{array}$ & $\mathrm{DE}$ & Min & Máx \\
\hline Q1 Calidad de vida & 2,41 & 0,66 & 1 & 3 \\
\hline Q2 Salud General & 2,5 & 1,16 & 1 & 5 \\
\hline Q3 Dolor y disconfort & 2,91 & 1,24 & 1 & 4 \\
\hline Q4 Dependencia medicación & 3,25 & 1,24 & 1 & 4 \\
\hline Q5 Sentimientos positivos & 2,5 & 1,44 & 1 & 5 \\
\hline Q6 Espiritualidad & 3,33 & 0,98 & 2 & 5 \\
\hline Q7 Pensamiento y memoria & 2,66 & 0,98 & 2 & 5 \\
\hline Q8 Seguridad & 2 & 1,27 & 1 & 5 \\
\hline Q9 Ambiente físico & 2 & 0,95 & 1 & 4 \\
\hline Q10 Energía y fatiga & 2,83 & 1,4 & 1 & 5 \\
\hline Q11 Imagen corporal & 2,74 & 1,35 & 1 & 5 \\
\hline Q12 Finanzas & 1,83 & 1,4 & 1 & 5 \\
\hline Q13 Acceso a información & 1,75 & 1,21 & 1 & 5 \\
\hline Q14 Actividades placenteras & 2,08 & 1,56 & 1 & 5 \\
\hline Q15 Movilidad & 4 & 1,53 & 1 & 5 \\
\hline Q16 Sueño y descanso & 1,83 & 1,33 & 1 & 5 \\
\hline Q17 Actividades cotidianas & 2,33 & 1,15 & 1 & 5 \\
\hline Q18 Capacidad para trabajar & 1,91 & 1,56 & 1 & 5 \\
\hline Q19 Autoestima & 2,25 & 1,21 & 1 & 5 \\
\hline Q20 Relaciones personales & 2,16 & 1,11 & 1 & 5 \\
\hline Q21 Actividad sexual & 1,83 & 1,26 & 1 & 5 \\
\hline Q22 Soporte social & 1,75 & 1,28 & 1 & 5 \\
\hline Q23 Condiciones vivienda & 1,33 & 1,15 & 1 & 5 \\
\hline Q24 Servicios de salud & 1,5 & 1,16 & 1 & 5 \\
\hline Q25 Transporte & 1,33 & 1,15 & 1 & 5 \\
\hline Q26 Sentimientos negativos & 3,33 & 1,66 & 1 & 5 \\
\hline
\end{tabular}

Fuente: elaboración propia. 
Tabla 3

Resultados cuestionario de calidad de vida (WHOQoL-Bref) de las MPL por dimensión.

\begin{tabular}{lcccc}
\hline Dimensiones WHOQoL-Bref & \multicolumn{2}{c}{ General $(\mathrm{n}=12)$} & Min & Máx \\
\hline Salud Física & M & DE & 17 & 13 \\
Psicológica & 42,75 & 22,05 & 10 & 29 \\
Relaciones Sociales & 42,25 & 22,39 & 0 & 75 \\
Ambiente & 31,66 & 25,56 & 0 & 94 \\
\hline
\end{tabular}

Fuente: elaboración propia.

\section{DISCUSIÓN}

Es importante tener en cuenta que los resultados obtenidos en esta investigación, a través de las evaluaciones antropométricas, de la condición física y calidad de vida, son de gran ayuda para conocer el estado de salud en el que se encuentran las reclusas del centro penitenciario de Concepción. Las MPL presentaron un IMC promedio en categoría de sobrepeso $(27,85 \pm 6,08)$, un ICC que muestra riesgo cardiovascular moderado $(0,85 \pm 0,05)$ y un resultado en saltabilidad bajo $(13,46 \pm 4,72)$ que, al contrastarlo con los resultados del estudio de Castro, Monsalves \& Pérez (2014) realizado en jóvenes chilenas sedentarias, donde se evalúan características antropométricas de riesgo cardiovascular y saltabilidad, podemos observar que presentan sobrepeso (25,44 \pm $5,52)$, bajo riesgo cardiovascular $(0,73 \pm 0,05)$ y un promedio de CMJ de 18,20 \pm 5,00; si bien el IMC se encuentra dentro de la misma categoría, presentan un menor riesgo cardiovascular y un puntaje más alto en CMJ, esto puede estar relacionado con que las jóvenes promedian una edad de 20,95 \pm 1,62, mientras que las reclusas, 39,58 \pm 15,30 años. Escobar (2018) detectó que, en Chile existen falencias en el ámbito de la salud física y mental de las reclusas, así como la necesidad de implementar programas de vida saludable, nutrición y alimentación. Chumlea et al. (1988) mencionan que la falta de movimiento, cambios en la dieta y reducción de la actividad física pueden llevar hacia la obesidad y la variación del peso corporal; estas características no sólo se presentan en la población recluida en centros penitenciarios, sino también en la población general femenina chilena con un 33,75\% de obesidad y un 36,4\% de sobrepeso ( $M i-$ nisterio de Salud [MINSAL], 2017). En función de la prueba de salto, Buskirk \& Taylor (1957) mencionan que el exceso de adiposidad incrementa el costo metabólico de actividades físicas, del movimiento de la masa total del cuerpo, concluyendo que mientras mayor sea la masa grasa, menor será la potencia de salto.

En la prueba de flexibilidad, las mujeres obtienen un resultado promedio categorizado como muy malo $(35,91 \pm 11,61)$, al compararlo con un estudio donde se midió la aptitud física en personas adultas, se obtuvo para las mujeres en flexibilidad de tronco obtuvieron un rendimiento regular $(34,41 \pm 5,75$ ) (Rojas et al., 2016). En otro estudio de personas adultas de trabajo administrativo, se midió la flexibilidad en mujeres promediando 20,24 土 10, lo que se clasificó como bajo la media de los valores normativos y se llegó a la conclusión que en conjunto con otros factores afectan el desempeño laboral y la calidad de vida (Xavier et al., 2016). De acuerdo a los antecedentes, es importante considerar que "la flexibilidad es uno de los componentes de la aptitud física relacionada con la salud y el rendimiento físico" (Chaves et al., 2016, p. 256) y que los ejercicios orientados a la flexibilidad, además de ayudar a mejorar los rangos de movimiento de las articulaciones y la elasticidad de los grupos musculares, evita la atrofia por desuso, aliviando molestias osteomusculares que, en muchas ocasiones, son producidas por falta de actividad física (Durón, 2011).

La aptitud cardiorespiratoria promedio de las MPL evaluada a través del test del escalón, fue categorizada como pobre $(28,32 \pm 5,48)$, al compararlo con el estudio de Díaz \& Saavedra (2008) donde se 
midió la condición física en adultos chilenos a través del test de $2 \mathrm{~km}$ de caminata, encontraron que del total de la muestra para el sexo femenino, un 18\% presentaban buena condición física, el 22,9\% presentaba condición física regular y el 59\% mala condición física, además mostró que a medida que aumentaba la edad disminuía el rendimiento. En un estudio que buscaba favorecer la condición física en mujeres adultas se encontró que la mayoría de la población evaluada no mostró resultados satisfactorios en la prueba de resistencia cardiorespiratoria categorizando los resultados en malo $(33,3 \%)$ y pobre $(33,3 \%)$ (León \& Céspedes, 2014). Es importante conocer el nivel de condición física y más específicamente la capacidad aeróbica, ya que "la aptitud cardiovascular tiene importantes implicaciones para la salud" (Welk et al., 2011, p. 111). La práctica de actividad física no sólo consigue beneficios en la dimensión física, sino que también contribuye a mejorar la condición psíquica y social de las personas (González, 2004). Por tanto, es necesario para la promoción de la salud, la implementación de programas de actividad física que tengan como objetivo cambiar los estilos de vida para así mejorar su calidad de vida (Vidarte et al., 2011).

En los resultados del cuestionario WHOQOLBref de las MPL chilenas se obtienen puntajes que los posicionan en el percentil más bajo (P5) de acuerdo a los valores de referencia de la población adulta chilena (Urzúa \& Caqueo, 2013), obteniendo un puntaje promedio más bajo en la dimensión ambiente $(19,25+-25,56)$ y el promedio más alto en la dimensión física $(42,75$ + -19,4). Los resultados de un estudio con mujeres reclusas donde se les aplicó el mismo instrumento (WHOQoL-Bref) los puntajes

\section{CONCLUSIÓN}

El estudio permite concluir que las MPL se encuentran con sobrepeso, riesgo cardiovascular moderado y una baja condición física. La percepción de la calidad de vida general es baja y en todos los dominios del cuestionario los resultados se encuentran en el percentil más bajo. promedios de la calidad general de vida de las reclusas fueron bajos (46), el dominio con la media más alta fue el físico y el más bajo fue el de medio ambiente (Ferreira \& Fernandes, 2020), al comparar ambos estudios tienen resultados muy similares y con las mismas tendencias, pero las reclusas chilenas tienen puntajes promedios más bajos que las reclusas brasileñas. Por otra parte, en un estudio donde las mujeres eran productivamente activas, la calidad de vida fue considerada como buena $(63,7)$, donde el dominio con peor puntuación media fue el ambiente $(54,6)$ (Rodrigues \& Fernandes, 2017). De acuerdo a lo anterior, se puede desprender que la prisión influye negativamente en la percepción de la calidad de vida de las mujeres (Ferreira \& Fernandes, 2020). Para Urzúa \& Caqueo (2012) el resultado final de la evaluación de la calidad de vida se encuentra influenciado por una diversidad de factores que intervienen en la percepción de una persona, "constituyéndose en un abordaje multidimensional, ya que se conjugan diversas valoraciones que hace el propio sujeto sobre las distintas aristas de su vida y de qué manera percibe bienestar o malestar referente a ellas" (p.65)

Este estudio tuvo como limitación la cantidad de reclusas participantes de la investigación, además que se desarrolló sólo en un centro penitenciario, el tiempo fue limitado para la recolección de datos de acuerdo a las restricciones propias de un centro penitenciario en función del ingreso de materiales de evaluación y cantidad de evaluadores. Sin embargo, la investigación permite obtener nueva información acerca de la realidad de las mujeres dentro del centro penitenciario en la ciudad de Concepción, Chile.

La identificación de un perfil de estado nutricional, riesgo cardiovascular, condición física y calidad de vida puede orientar acciones de intervención en el área de la actividad física para mejorar la salud y calidad de vida de las mujeres privadas de libertad del país. 
Se recomienda realizar estudios para evaluar otros parámetros de la condición física, incorporando información asociada a ECNTy mayores antecedentes relacionados con la salud.

\section{REFERENCIAS BIBLIOGRÁFICAS}

American College of Sports Medicine. (2005). Manual ACSM para la valoración y prescripción del ejercicio. Paidotribo.

Barra, E. (2003). Influencia del estado emocional en la salud física. Terapia Psicológica, 27(1), 5560. https://www.researchgate.net/profile/Enrique_Barra_Almagia/publication/235760005_Influence_of_emotional_state_on_physical_health_Influencia_del_estado_emocional_en_la_salud_fisica/links/09e4151339d8956e18000000.pdf

Bobes, J., Portilla, M., Bascarán, M., Saiz, P., \& Bousoño M. (2004). Banco de instrumentos básicos para la práctica de la psiquiatría clínica (3a ed.). Ars Médica.

Bosco, C. (1996). Aspectos fisiológicos de la preparación física del futbolista. Paidotribo.

Buskirk, E., \& Taylor, H. (1957). Maximal oxygen intake and its relation to body composition, with special reference to chronic physical activity and obesity. Journal of Applied Physiology, 2, 72-78. https://journals.physiology.org/doi/pdf/10.1152/jappl.1957.11.1.72

Cárdenas, A. (2011). Mujer y cárcel en Chile. https://www.icso.cl/wp-content/uploads/2017/03/Mujer-y-carcel.pdf

Casas, L., Cordero, R., Espinoza, O., \& Osorio, X. (2005). La perspectiva de género en la defensa de mujeres en el nuevo sistema procesal penal chileno: un estudio exploratorio. http://www.corteidh.or.cr/tablas/r17942.pdf
Castro-Sepúlveda, M., Monsalves, M., \& Pérez, C. (2014). Relación entre la altura de salto y variables antropometricas de riesgo cardiovascular en jóvenes sedentarias. Revista de Educación Física, 30(3), 1. https://gse.com/relacion-entre-altura-de-salto-y-variables-antropometricas-de-riesgo-cardiovascular-en-jovenes-sedentarias-1729-saC57cfb27244b8a

Celis-Morales, C., Salas, C., Leiva, A., Garrido-Méndez, A., \& Díaz-Martínez, X. (2017). Costo económico asociado a la inactividad física en Chile. Revista Médica de Chile, 145, 10891092. https://scielo.conicyt.cl/pdf/rmc/v745n8/0034-9887-rmc145-08-1091.pdf

Chaves, T., Balassiano, D., \& Araujo, C. (2016). Influencia do hábito de exercício na infancia e adolescencia na flexibilidade de adultos sedentários. Revista Brasileira de Medicina do Esporte, 22(4), 256-260. https://www.scielo.br/pdf/rbme/v22n4/1517-8692-rbme-2204-00256.pdf

Chumlea, W., Garry, P., Hunt, W., \& Rhyne, R. (1988). Distribution of Serial Changes in Stature and Weight in a Healthy Elderly Population. Human Biology, 60(6), 917-925. http://www.jstor.org/stable/41464085

Comité de Derechos Económicos, Sociales y Culturales. (2000). El derecho al disfrute del más alto nivel posible de salud. https://www.acnur.org/fileadmin/Documentos/BDL/2001/1451.pdf 
Díaz, E., \& Saavedra, C. (2008). Evaluación de la condición física en adultos chilenos. http://www.mindep.cl/wp-content/uploads/2016/06/03-Desarrollo-y-validacion-de-una-tecnica-para-la-evaluacionde-la-condicion-fisica-2006.pdf

Durón, H. (2011). La actividad física y el adulto mayor: un factor de salud y calidad de vida. Revista Facultad Ciencias Médicas, 8(1) 61-65. http://65.182.2.244/RFCM/pdf/2011/pdf/RFC MVol8-7-2017.pdf\#page=59

Escobar, D. (2018). Análisis al derecho a la salud de mujeres privadas de libertad. [Tesis Licenciatura, Universidad de Chile]. http://reposito-

rio.uchile.cl/bitstream/handle/2250/159428/ An\%C3\%A7lisis-del-derecho-a-la-salud-demujeres-privadas-de-libertad.pdf?sequence=1\&isAllowed=y

Ferreira, M., \& Fernandes, R. (2020). Mulheres detentas do Recife-PE: saúde e qualidade de vida. Escola Anna Nery, 24(4), 1-12. https://www.scielo.br/pdf/ean/v24n4/en_1414-8145-ean-244-e20200062.pdf

Gendarmería de Chile. (2018). Compendio Estadístico Penitenciario 2018. https://html.gendarmeria.gob.cl/doc/estadisticas/Compendio_Estadistico_Penitenciario_2018.pdf

Gómez, A. (2007). Salud física y salud mental. Farmacia Abierta, 27(7), 53-56. https://www.elsevier.es/es-revista-farmacia-profesional-3pdf-13108595

González-Jurado, J. (2004). La actividad física orientada a la promoción de la salud. Escuela Abierta: Revista de Investigación Educativa, (7),73-96. https://dialnet.unirioja.es/servlet/articulo?codigo $=1065700$
Hernández, R., Fernández, C., \& Baptista, M. (2014). Metodología de la Investigación ( $6^{a}$ ed.). Mc Graw-Hill.

Instituto Nacional de Derechos Humanos. (2012). Informe anual 2012 situación de los derechos humanos en Chile. https://bibliotecadigital.indh.cl/bitstream/handle/123456789/296 /informe-anual-2012.pdf?sequence=1\&isAllowed=y

León, A., \& Céspedes, Y. (2014). Programa de ejercicios que favorecer la condición física en mujeres adultas. EFDeportes, 19(199),1. https://www.efdeportes.com/efd199/favorecer-la-condicion-fisica-en-mujeres-adultas.htm

Martínez, E. (2002). Pruebas de aptitud física. Paidotribo

Mei, Z., Grummer-Strawn, L., Pietrobelli, A., Goulding, A., Goran, I., \& Dietz, W. (2002). Validity of body mass index comparated with other body-composition screening indexes for assessment of body fatness in children and adolescents. American Journal of Clinical Nutrition, 75, 978-985. https://pubmed.ncbi.nlm.nih.gov/12036802/

Ministerio de Salud. (2008). Guía clínica examen de medicina preventiva. https://www.minsal.cl/por-

tal/url/item/73b3fce9826410bae04001011f01 $7 f 7 b . p d f$

Ministerio de Salud. (2017). Encuesta nacional de salud 2016-2017. https://www.minsal.cl/wpcontent/uploads/2017/11/ENS-2016-17_PRIMEROS-RESULTADOS.pdf

Organización de las Naciones Unidas para la Alimentación y la Agricultura (FAO) y Organización Panamericana de la Salud (OPS). (2017). Panorama de la seguridad alimentaria y nutricional en América Latina y el Caribe. http://www.fao.org/3/CA2127ES/CA2127ES.p df 
Organización Mundial de la Salud. (1996). WHOQOLBREF Introduction, administration, scoring and generic version of the assessment. https://www.who.int/mental_health/media/en/76.pdf?ua=1

Organización Mundial de la Salud. (2010). Global recommendations on physical activity for health.

https://apps.who.int/iris/bitstream/handle/1 0665/44441/9789243599977_spa.pdf;jsessio-

nid=8A5C7E9BE5E3E36C032871C6097042 OD? sequence=1

Otzen, T., \& Manterola, C. (2017). Técnicas de muestreo sobre una población de estudio. International Journal of Morphology, 35(1), 227232. https://scielo.conicyt.cl/pdf/ijmorphol/v35nt/art37.pdf

Robles-Espinoza, A., Rubio-Jurado, B., De la RosaGalván, E., \& Nava-Zavala, A. (2016) Generalidades y conceptos de calidad de vida en relación con los cuidados de salud. El Residente, 17(3), 120-125. https://www.medigraphic.com/pdfs/residente/rr2016/rr163d.pdf

Rodrigues, M., \& Fernandes, R. (2017). Calidad de vida y morbilidad referida a mujeres productivamente activas. Enfermería Global, (46), 246257.

http://scielo.is-

ciii.es/pdf/eg/v16n46/1695-6141-eg-16-4600246.pdf

Rojas-Laverde, D.,Arce-Varela, E., Suárez-Orozco, Y., Moya-Castro, D., Barrantes-Segura, M., Cambronero-Steller, F., \& Molina-DeBernardi, M. (2016). Diferencias según sexo en la aptitud física de personas adultas que acuden a un centro urbano de recreo de Costa Rica. Revista Movimiento Científico, 10(1), 39-53. https://revmovimientocientifico.ibero.edu.co/article/view/mct.10104/882
Rosa-Guillamón, A., García-Cantó, E. Rodríguez-García, P., \& Pérez, J. (2017). Condición Física y calidad de vida en escolares de 8 a 12 años. Revista de la Facultad de Medicina, 65(1), 37-42. https://doi.org/10.15446/revfacmed.v65n1.59634

Sanhueza, G., Brander, F., \& Reiser, L. (2019). Encarcelamiento femenino en Chile calidad de vida penitenciaria y necesidades de intervención. Revista de Ciencias Sociales, 32(45), 119-745.

http://dx.doi.org/10.26489/rvs.v32i45.5

Urzúa, A., \& Caqueo-Urízar, A. (2012). Calidad de vida: Una revisión teórica del concepto. Terapia Psicológica, 30(1), 61-71. https://scielo.conicyt.cl/pdf/terpsicol/v30n7/art06.pdf

Urzúa, A., \& Caqueo-Urízar, A. (2013). Estructura factorial y valores de referencia del WHOQOlBref en población adulta chilena. Revista Médica de Chile, 147(12), 1547-1554. https://scielo.conicyt.cl/scielo.php?script=sci_arttext\&pid=S 0034-98872013001200008

Vargas, F. (Ed.). (2019). Informe anual sobre derechos humanos en Chile 2019. http://www.derechoshumanos.udp.cl/derechoshumanos/images/InformeAnual/2019/12_La_institucionalidad_de_Derechos_Humanos_en_Chile.pdf

Vidarte, J., Vélez, C., Sandoval, C., \& Alfonso, M. (2011). Actividad física: estrategia de promoción de salud. Hacia la Promoción de la Salud, 6(1), 202-208. http://www.scielo.org.co/pdf/hpsal/vi6nt/v1 6nla14.pdf 
Welk, G., Laurson, K., Eisenman, J., \& Cureton, K. (2011). Development of youth aerobic-capacity standars using receiver operating characteristic curves. American Journal of Preventive Medicine, 47(2), 117-116. http://www.cooperinst.org/vault/2440/web/ files/791.pdf

World Health Organization (1998). WHOQOL user manual. https://www.who.int/mental_health/evidence/who_qol_user_manual_98.pdf

Xavier, I., Meneghini, L., Pereira, A., \& Monterrosa, A. (2016). Incomodidad Corporal, Carga Física y Nivel de Flexibilidad en Trabajadores del Sector Administrativo de una Institución de Enseñanza Superior en Florianópolis, del Sur de Brasil. Ciencia \& Trabajo, 18(57), 145149. http://dx.doi.org/10.4067/s071824492016000300145

Zaragoza, J. Serrano, E., \& Generelo, E. (2004). Dimensiones de la condición física saludable: evolución según edad y género. Revista Internacional de Medicina y Ciencias de la Actividad Física y el Deporte, 4(15), 204-221. http://coleporte.rediris.es/revista/revista15/artdimensiones.htm

\section{Dirección para correspondencia}

Mg. Yazmina Virginia Pleticosic Ramírez

Universidad San Sebastián

Concepción, Chile.

CP: 4080871

ORCID: https://orcid.org/0000-0003-1710-6663

Contacto:

yazminapleticosic@gmail.com

Recibido: 14-08-2020

Aceptado: 27-01-2021 